\title{
Controlando a utilização indiscriminada de tecnologias médicas e a escalada dos custos dos sistemas de saúde: a estratégia alemã
}

\author{
Controlling the indiscriminate use of medical \\ technologies and the rise in the costs of health \\ care: the German strategy
}

Ricardina Giovanna Pitelli da Guia

Departamento de Prática de Saúde Pública, Faculdade de Saúde Pública, Universidade de São Paulo. Av. Dr. Arnaldo 715, São Paulo, SP, 01246-904, Brasil.
Abstract In view of reforms implemented in health care systems in many countries, both developed or developing, and especially in view of the implementation of the Sistema Único de Saúde - SUS (Single Health System) in Brazil, the author presents some aspects of the reform process in the German Health Care System. Special attention is given to the various mechanisms employed in Germany to keep health care costs stable in the system in relation to the Gross Domestic Product (GDP), mainly through the adoption of measures to rationalize use of medical technologies. Some aspects related to the broad bargaining process between the Medical Boards and the funds providing health care services are discussed, with a view towards elaboration of parameters for assessing medical performance, particularly in relation to utilization of medical technological resources. Finally, the author briefly discusses the impact of such measures in producing changes in medical performance standards.

Key words Medical Technology; Health Care Systems; Medical Practice; Health Policy; Public Health

Resumo Com vistas às reformas que vêm sendo executadas nos Si stemas de Saúde dos mais diversos países do mundo desenvolvido ou em desenvolvimento, e, em especial, com vistas à implementação do Si stema Único de Saúde - SUS, a autora apresenta alguns aspectos do processo de reforma que vem sendo posto em prática no Si stema de Saúde da Alemanha. Atenção especial é dada aos di versos mecanismos ali utilizados, no senti do de manter os gastos do Sistema de Saúde estáveis em relação ao Produto Interno Bruto (PIB), principalmente através da adoção de medi das racional izadoras do uso de tecnologias médi cas. Discutem-se aspectos rel ati vos ao amplo processo de negoci ação estabel eci do entre as Associ ações de M édi cos e os Fundos provedores de cui dados à saúde, no senti do de viabilizar a elaboração de parâmetros para avaliação da performance médica, parti cularmente em relação à uti lização de recursos médi co-tecnológi cos. E, finalmente, são el aboradas al gumas breves considerações acerca do i mpacto de tais medi das em propiciar mudanças nos padrões de atuação médica.

Palavras-chave Tecnologia Médica; Sistemas de Saúde; Prática Médica, Política de Saúde; Saúde Pública 


\section{Introdução}

O Sistema de Saúde da Alemanha, à semelhança daqueles de vários outros países do mundo, vem passando por transformações (Organizacion Mundial de la Salud, 1992; Reinhardt, 1994), a maioria das quais destina-se a promover e incentivar uma utilização mais eficiente e racional dos recursos disponíveis, sem provocar alterações significativas na estrutura organizacional e na forma de financiamento do Sistema, criado no final do século passado, em 1883, por Bismark. Uma característica notória do Sistema de Saúde alemão tem sido a capacidade de manter seus gastos estáveis, em torno de $8,7 \%$ do PIB, durante os anos 80 , concomitantemente à preservação, e até mesmo ampliação (com a incorporação da Alemanha Oriental), do acesso universal da população (80 milhões de habitantes em 1993) aos serviços básicos de saúde. No entanto, nos primeiros anos da década de 90 , um aumento nos gastos gastos do sistema, os quais chegam a $9,1 \%$ do PIB, em 1991 (Henke, 1994), concorrem para a implementação de medidas racionalizadoras e controladoras, como veremos adiante.

A Alemanha caracteriza-se por ter um Sistema de Saúde descentralizado, corporativista e pluralista, financiado por contribuições compulsórias sobre os salários (em média 13,42\% dos vencimentos brutos, sendo metade paga pelo empregado e metade pelo empregador), independentemente do fato de o trabal hador pertencer ao setor público ou privado (Schulenburg, 1994). O Estado apresenta papel inexpressivo como fonte de financiamento para o Sistema, mas dispõe de forte papel regulador sobre o mesmo. Sua fonte de financiamento o torna particularmente sensível a oscilações no nível de emprego e na estrutura do mercado de trabalho, onde setores que remuneram melhor seus trabalhadores têm menos probabilidade de apresentar Fundos de Doença com problemas de caixa que os setores com faixas salariais mais baixas. Até os anos 70, as taxas de desemprego alemãs eram baixas e estáveis, mas, a partir da segunda metade dos anos 80 , o desemprego aumentou em $11 \%$ para os trabal hadores denominados "blue-collar" (faixas salariais mais baixas) e $6 \%$ para os "white-collar" (faixas salariais mais altas), problemas estes agravados pela acentuada mudança na estrutura etária da população, assim como pelo alto índice de desemprego entre as pessoas que tinham de 55 a 60 anos (Wysong, 1990). E veremos adiante que, no final dos anos 70, a Alemanha passa a desenvolver e implementar, sistematicamente, os mais variados mecanismos com o objetivo não apenas de diminuir os gastos do Sistema, como também de melhorar a qualidade dos serviços prestados.

O Sistema é organizado em torno de Fundos de Doença, instituições de caráter privado não-lucrativo, cuja filiação é compulsória para determinados segmentos da população, de acordo com a faixa salarial a que pertencem, a qual é reajustada anualmente mediante dispositivo legal. Tais Fundos apresentam caráter meramente provedor, e não executor de serviços, comprando-os, mediante contratos, das diversas instituições prestadoras, sejam elas hospitais, laboratórios, grupos de médicos, etc.

Em 1994, havia 1.297 Fundos de Doença na Alemanha (Schulenburg, 1994), sendo que 1.091 local izavam-se na região oeste e 206 na região leste (antiga Alemanha Oriental). Tais Fundos estabelecem-se a nível dos Länder (Estados) ou em função de determinadas empresas, grupos de empresas, ou ramos de atividade econômica e atuam, geralmente, com abrangência regional, exceção feita às grandes empresas que, freqüentemente, dispõem de um único Fundo de Doença com abrangência nacional. São entidades autônomas, gerenciadas por representantes de empregados e empregadores, el eitos pela população segurada. Todo Fundo de Doença ou Seguro-Saúde privado deve oferecer a seus filiados um "pacote básico" de serviços, cujo conteúdo é determinado por instrumento legal e fiscalizado pelo governo, vigorando em todo o território nacional, independente da região ou das características da população segurada. A contribuição compulsória aos Fundos de Doença é relacionada direta e exclusivamente à capacidade de pagar, isto é, ao salário, independentemente da idade, sexo, número de dependentes ou situação de saúde do contribuinte ou filiado. Já os prêmios dos Seguros-Saúde privados têm seu valor relacionado a idade, número de dependentes, estado de doença, etc. do segurado, apesar de haver regulamentação governamental no tocante à parcela do prêmio referente ao "pacote básico", cujo preço é controlado e atualizado anualmente.

A filiação aos Fundos de Doença é compulsória para todo aquele que tenha vencimentos inferiores a um teto estabelecido por lei, sendo que, em 1993, tal valor era de US\$ 3.253 mensais (Müller, 1994). Para os que têm vencimentos acima deste valor, a filiação aos Fundos de Doença é optativa, pois o indivíduo pode escoIher por associar-se a um dos vários SegurosSaúde privados que atuam na Alemanha (em 1993, havia 64 Seguros-Saúde privados, a maioria de caráter não-lucrativo, sendo que os 23 
Seguros lucrativos detinham $46 \%$ do mercado) (Schulenburg, 1994). Os profissionais liberais e os trabalhadores autônomos geralmente associam-se a Fundos de Doença denominados Substitutivos, os quais, via de regra, oferecem amplos serviços adicionais ao "pacote básico" obrigatório e são gerenciados apenas por representantes dos filiados.

A cobertura aos desempregados e deficientes é garantida através de contribuições aos Fundos realizadas pelo Estado, sendo que os aposentados são distribuídos entre os Fundos e pagam uma taxa proporcional a sua aposentadoria como contribuição ao Fundo de Doença que escolheram, enquanto os trabalhadores da ativa pagam uma taxa adicional aos Fundos para ajudar a financiar o seguro dos aposentados, cujas taxas de contribuição cobrem apenas $41 \%$ dos custos da atenção (Schulenburg, 1994). Assim, o Sistema garante a cobertura assistencial a toda população, sendo que $88 \%$ desta encontram-se filiados compulsoriamente a um dos Fundos de Doença e 12\% dispõem de Seguro-Saúde privado.

O Sistema permite que os Fundos de Doença se estabeleçam através de mecanismos de competição pela adesão da população a ser se gurada, através da oferta de serviços adicionais ao pacote básico ou planos complementares ao mesmo, sendo que, desde 1993, o cidadão pode optar por trocar o Fundo ao qual está filiado por outro que julgue mais adequado, uma vez por ano. Nenhum Fundo de Doença pode negar-se a receber uma filiação, mesmo que demandadas por pessoas de alto risco para determinadas doenças ou de pacientes portadores de doenças crônicas, assim como não pode negar-se a receber filiados de baixa renda. Assim, a competitividade entre os Fundos nem sempre se estabelece na prática, pois, uma vez dependendo da faixa salarial da população segurada, os Fundos de Doença que cobrem a população de baixa renda ou de alto risco, freqüentemente, não dispõem de recursos para adentrar tal competição: conseguindo oferecer apenas o "pacote básico", acabam por afugentar filiados com maior renda, os quais procuram Fundos que ofereçam mai or diversidade de serviços complementares ou adicionais, como "spas" após cirurgias ou atividades de recreação nas férias, etc. Por outro lado, a obrigatoriedade de prover um "pacote básico" de serviços vinha conduzindo alguns Fundos a aumentar a quota de contribuição sobre o salário do filiado como medida para garantir a oferta de tal "pacote" (enquanto alguns Fundos demandavam uma contribuição de 10,8\% sobre o salário do trabalhador, outros chegavam a demandar 16,4\%) (Schulenburg, 1994), gerando profundas desigualdades entre os Fundos e, conseqüentemente, ferindo o princípio de solidariedade que, há décadas, tem orientado a organização do Sistema de Saúde Alemão. A competição, mais do que melhorar a qualidade dos serviços prestados, aumentava e incentivava a estratificação entre os Fundos e a extensão dos serviços, acentuando as diferenças entre pobres e ricos e inflacionando os custos dos Sistema.

Vários fatores se sobrepunham, acentuando as diferenças entre os Fundos "pobres" e "ricos", uma vez que os trabalhadores com menor renda costumam ser aqueles que mais consomem serviços de saúde, assim como freqüentemente dispõem de condições de trabalho menos salubres ou com maior risco. Por outro lado, segundo Powell (1994), principalmente a partir dos anos 70, os Fundos que dispunham de maiores recursos, como os Substitutivos e os Seguros-Saúde privados, começaram a pressionar pela oferta de serviços mais caros e sofisticados, incorporando aceleradamente novas tecnologias médicas, sob o argumento que seus associados estavam dispostos a pagar por tais benefícios. Tal tendência criava novas expectativas para a população segurada, a qual pressionava para a inclusão de novos serviços ao "pacote básico" dos Fundos mais pobres, inflacionando os custos do Sistema e tornando muitos Fundos deficitários. Assim, em 1977, entra em vigor uma nova legislação, destinada a conter os gastos e a racionalizar a utilização de determinados procedimentos médico-tecnológicos pelos serviços de saúde alemães, denominada "Lei de Contenção de Custos" (LCC). Após sua introdução, em janeiro de 1977, a LCC é responsabilizada pela queda nos gastos de sistema, os quais, em 1976, haviam sido em torno de $8,7 \%$ do PIB, e passam, em 1977, a 5,7\% (Roy, 1993).

Em síntese, o Sistema de Saúde alemão apresenta um modelo corporativista, onde os Fundos de Doença e as Associações de Médicos operam como instituições semi públicas, as quais têm autoridade legal para financiar, organizar e distribuir serviços de saúde à população segurada.

\section{O sistema de atenção ambulatorial}

O Sistema de Saúde al emão caracteriza-se por diferenciar claramente atenção ambulatorial de atenção hospitalar, assim como não permite que os médicos atuem indistintamente nos dois setores: ele atua ou em hospitais, ou no 
sistema ambulatorial, sendo que, apenas com raríssimas exceções e em condições especiais, é permitido a um médico atuar concomitantemente nos setores hospitalar e ambulatorial.

O setor ambulatorial alemão é composto por médicos que atuam de forma autônoma e privada, individualmente ou em grupos, prestando serviços aos Fundos de Doença mediante contratos. O contrato com os Fundos, no entanto, não é estabelecido individualmente por cada médico ou grupos de médicos e sim de forma coletiva: para atuarem junto aos Fundos, os médicos alemães devem estar filiados às Associações Regionais de Médicos (havia 19 em todo o país, em 1993) (Roy, 1993), as quais, por sua vez, congregam-se na Associação Nacional de M édicos, sendo que a nenhum médico pode ser vetado o direito de filiar-se a tais Associações. Cada Associação Regional negocia contratos coletivos com um ou mais Fundos, podendo estabelecer ou negociar preços diferentes com Fundos diferentes, mesmo que relacionados à execução de um mesmo procedimento ou serviço. Via de regra, os Seguros-Saúde privados pagam honorários médicos duas a três vezes maiores que os Fundos de Doença Compulsórios pela realização de um mesmo procedimento, nas mesmas circunstâncias, o que tem sido apontado como uma das causas da presença de uma maior fila de espera por consulta entre a população segurada pelos Fundos de Doença em relação à coberta pelos Seguros-Saúde privados.

O sistema ambulatorial alemão, desde sua criação, estabelecia os pagamentos em função do número de procedimentos realizados ("fee for service"), o que vinha aumentando aceleradamente os gastos do setor, até que, em 1977, com a LCC, os Fundos de Doença são proibidos de apresentar déficit orçamentário, sob pena de intervenção legal do governo, isto é, os Fundos não podiam gastar mais do que arrecadavam com seus filiados. Tal medida incorreu na necessidade de calcular-se o preço dos procedimentos médicos a serem pagos ao setor ambulatorial em função do montante de recursos disponíveis para tal, e não mais com base em valores preestabelecidos para cada procedimento.

As medidas estabelecidas em 1977, com a “Lei de Contenção de Custos" (LCC), demandavam que se calculasse anualmente uma margem de risco para procedimentos tecnológicos, especificando o montante de recursos que poderia ser consumido com tais serviços. Estabelecia também metas racionalizadoras a serem alcançadas, através do desenvolvimento de um fator de correção que estimava a "taxa de mudança tecnológica" para cada área específica de serviços, com base nos dados disponíveis sobre as mudanças tecnológicas nos anos anteriores e pesquisas em andamento.

O pagamento dos honorários médicos é realizado com intermediação das Associações de Médicos, e não diretamente pelos Fundos de Doença, as quais calculam e reajustam o preço de cada procedimento médico, trimestralmente, com base na arrecadação do trimestre anterior, sendo que eventuais distorções são corrigidas no último trimestre do ano. Assim, quanto menor a quantidade de recursos recebida, menor será o preço pago por procedimento realizado, o contrário se dando quando ocorre a realização de um menor número de procedimentos ou uma maior disponibilidade de recursos. Também compete às Associações de Médicos monitorar a qualidade dos serviços prestados por seus associados, assim como fiscalizar a veracidade das faturas apresentadas, de forma a evitar a utilização inadequada dos recursos repassados pelos Fundos, os quais, por sua vez, também dispõem de variados instrumentos para monitorização da qualidade e eficiência dos serviços que Ihe são prestados pelas diversas instituições e serviços de saúde.

A associação entre volume de procedimentos realizados e rendimentos médicos se explica pelo fato de os serviços de apoio diagnóstico pertencerem, via de regra, aos médicos que atuam no sistema ambulatorial, sendo responsáveis, em 1986, por 1/ 3 da renda dos médicos generalistas (Brenner, 1994) . Dentro do Sistema alemão, os exames de análises clínicas são classificados (Müller, 1994) em três categorias, de acordo com a complexidade dos procedimentos necessários a sua realização, sendo que tal tipologia apresenta uma relação direta com a estrutura demandada pelo laboratório que procede a tais análises.

Assim, os exames são classificados em:

- Exames para investigações básicas: contemplam a realização de urina I, hemograma, glicemia, etc. Podem ser realizados por médicos generalistas, assim como podem ser solicitados por qualquer médico;

- exames para investigações gerais: são exames metodologicamente simples, porém mais sofisticados que os básicos, como, por exemplo, dosagens enzimáticas, tais como TGO, TGP, etc. Também podem ser realizados pelos médicos generalistas e solicitados por qualquer médico;

- exames para investigações especiais: inclui testes laboratoriais metodologicamente mais complexos, como exames imunológicos, sorológicos e bacteriológicos. Tais exames só po- 
dem ser solicitados por especialistas, assim como só podem ser operacionalizados por médicos especialistas em patologia clínica.

Com base na qualificação anterior, os laboratórios de análises clínicas são classificados nas seguintes categorias:

- Laboratórios de Médicos Generalistas: são laboratórios pertencentes aos médicos generalistas (MG) e encontram-se acoplados ao sistema ambulatorial de atenção básica. Estimavase que, em 1993, cerca de 7.000 a 10.000 M Gs possuíssem seu próprio laboratório, os quais, via de regra, encontram-se equipados para proceder apenas a investigações básicas e gerais (Müller, 1994).

- Laboratórios de Grupo: primariamente destinam-se a uma Associação de M édicos Generalistas, clínicos ou outros especialistas, com o objetivo de garantir-Ihes testes eficientes, confiáveis e rápidos. Geralmente atendem a associações de, mais ou menos, 200 médicos, sendo que alguns laboratórios supra-regionais têm milhares de médicos associados, atendendo entre 800 e 1.000 pacientes por dia e procedendo de 4.000 a 5.000 exames diários (Müller, 1994). Geralmente realizam apenas investigações básicas e gerais.

- Laboratórios de Institutos: existiam por volta de 250 destes laboratórios na Alemanha em 1993 (Müller, 1994), os quais devem ser dirigidos e operacionalizados por médicos patologistas clínicos (cujo período de especialização, após a graduação, demanda cinco anos). Tais laboratórios concentram suas atividades na realização de investigações especiais.

- Laboratórios de Hospitais: havia por volta de 3.000 destes laboratórios em 1993 (Müller, 1994), sendo que grandes hospitais têm vários laboratórios, os quais, geralmente, são capacitados a realizar investigações básicas, gerais e especiais. Pequenos hospitais geralmente têm laboratórios encabeçados por clínicose, conseqüentemente, podem realizar apenas investigações bási cas e gerais. Os custos dos procedimentos laboratoriais em hospitais são incluídos nos preços das diárias de internação e não são calculados separadamente, à exceção dos pacientes particulares, caso em que os custos são calculados com base nas características de cada exame realizado.

Deste modo, quando um determinado Fundo apresenta tendências deficitárias inicia-se amplo processo de negociação entre as partes envolvidas - associações de médicos, hospitais, laboratórios e gestores do Fundo de Doença deficitário - na tentativa de estabelecer mecanismos para contenção de gastos, sendo que tal processo de negociação tem sido mais efeti- vo que a intervenção legal governamental "em si"; via de regra, quando a intervenção se dá, as propostas de solução já se encontram negociadas e implementadas entre as partes, como medida para evitar ingerências externas na gestão autônoma dos Fundos.

Tal estratégia tem dificultado aos médicos de ambulatório conhecerem previamente a quantidade de recursos destinada aos serviços que prestaram, pois a quantia a ser paga por cada procedimento tem uma relação indireta com o volume total de serviços executados, dependendo do montante de recursos disponível para financiar tais serviços. Deste modo, a cada três meses, as Associações de Médicos e os Fundos reavaliam o preço de cada procedimento específico, sendo que, quanto maior for o volume de procedimentos executados, menor será o preço unitário a ser pago por cada um, uma vez que o Sistema não pode apresentar déficit. Tal mecanismo, longe de incentivar a realização de um número menor de procedimentos como estratégia para aumentar seu preço unitário, tem incorrido num aumento no número de procedimentos realizados, pois os médicos tentam garantir uma "renda mínima" realizando o máximo possível de procedimentos.

O médico generalista se apresenta como a porta de entrada do Sistema de Saúde Alemão, sendo que, somente por sua indicação ou encaminhamento, o paciente pode consultar-se com um especialista ou internar-se em um hospital, salvo em situações de emergência. Apesar de vir implementando inúmeras medidas racionalizadoras para contenção de gastos, - Sistema não estabelece limites ou restrições de qualquer natureza em relação ao número de consultas com médicos generalistas, os quais, mediante suas prescrições de medicamentos, solicitações de exames e encaminhamentos para internação ou especialistas, direcionam a demanda por todos os demais serviços e níveis de atenção dentro do Sistema. Segundo Powell (1994), havia forte tendência dentro do sistema ambulatorial em se negociarem altos preços para novas tecnologias e exames diagnósticos, enquanto procedimentos que demandavam um maior consumo de tempo ou que eram menos lucrativos, como os relacionados a componentes de forte contato pessoal entre médico e paciente, eram remunerados com valores menores. Para ele, tal padrão encorajava a ampla utilização de caros serviços tecnológicos e diminuía o incentivo a exames clínicos, consultas e orientações preventivas, conduzindo a uma supervalorização de determinadas especialidades como radiologia e análises clínicas, 
introduzindo novos padrões de cuidados e causando inflação nos gastos do sistema.

Como conseqüência, em 1989, em pleno processo de unificação das duas Alemanhas, novas medidas racionalizadoras entram em vigor através da "Lei para Reforma do Sistema de Saúde" (LRSS), a qual estabelece co-pagamento (alguns de até 50\%) para vários procedimentos (incluindo hospitalização e próteses odontológicas) e medicamentos (mediante classificação de sua utilidade ou necessidade para determinadas doenças ou grupos de doenças), aumenta os prêmios dos Seguros-Saúde privados, estabelece pagamento com reembolso posterior para vários procedimentos que até então não demandavam qualquer transação financeira direta entre prestador e paciente, diminui a cobertura para cuidados odontológicos e amplia a cobertura a cuidados preventivos, entre outras medidas.

As medidas mais radicais da LRSS referiamse aos medicamentos, sendo que passaram a vigorar listas de preços de insumos farmacêuticos comparáveis e estabeleceram-se preços fixos para medicamentos cujos preços subiam mais que a inflação: o paciente só era reembolsado pelo Fundo no montante referente aqueles preços fixos, obrigando a indústria farmacêutica a rever seus preços sob o risco de perder faixas de mercado (Roy, 1993). Em janeiro de 1996, deverá ser criado um Instituto (Arzneimittelinstitut), subordinado ao Ministério da Saúde e composto por 11 especialistas, o qual implementará "normas" ("guidelines"), ou "regras para boa prescrição", para a utilização de medicamentos, estabelecendo os valores a serem cobrados como co-pagamento para determinadas drogas e, também, os limites para os preços dos medicamentos comercializados na Alemanha.

Em janeiro de 1993, entrou em vigor nova legislação, denominada Gesundheitsstrukturgesetz (GSG), estabelecendo várias mudanças a serem implementadas a médio e longo prazo, as quais pretendiam ser mais abrangentes que as até então implementadas. A principal delas, e introdutória para a unificação de todos os Fundos de Doença, constitui-se na criação, a partir de 1995, de um mecanismo para redistribuição de recursos financeiros entre os Fundos de Doença, denominado “Estrutura para Compensação de Riscos" (ECR), de maneira a diminuir as desigualdades na oferta e acesso aos serviços. Assim, os recursos serão redistribuídos entre os Fundos, de acordo com os riscos da população filiada, como faixa etária, número de dependentes, número de aposentados ou inválidos, portadores de doenças crônicas, etc.
A implementação da ECR deverá afetar as bases sobre as quais têm se estabelecido os mecanismos de competição entre os Fundos, os quais, provavelmente, não mais disporão de recursos adicionais abundantes para oferecer determinados serviços adicionais ao pacote básico, afetando principalmente a competitividade dos Fundos Substitutivos em relação ao setor privado.

Outra importante mudança dar-se-á em 1996, quando o pagamento por serviços ambulatoriais passará a ser feito, prioritariamente, com base em mecanismos de captação ( à semelhança do National Health Service - NHS, na Inglaterra), permanecendo apenas pequena parcela das atividades a ser paga por número de procedimentos realizados.

Em 1994, como uma tentativa de diminuir o número de exames laboratoriais realizados pelos MG, foi proposto o estabelecimento de uma quota de exames complementares por paciente atendido, independentemente do fato deste vir a utilizá-los ou não, sendo que apenas testes imunológicos ou sorológicos (solicitados apenas por especialistas, cujos pacientes não tinham que restringir-se a quaisquer quotas, pois estas só se destinavam aos MG) continuariam a ser remunerados como exames individuais, fora da quota. Tal medida suscitou muitas críticas e discussões entre os especial istas do setor, que acreditavam ser o pagamento por quota, o qual não demandava comprovação dos serviços prestados, incentivador da deterioração da qualidade dos laboratórios, diminuindo drasticamente a quantidade e a qualidade dos serviços prestados. Frente às críticas, as Associações de Médicos voltaram atrás e decidiram manter o pagamento por exame realizado, sendo que, no entanto, o número de exames será delimitado para cada grupo de médicos em função de sua especialidade e número de pacientes atendidos. Tais medidas, no entanto, ainda demandam aprovação dos Fundos, os quais, por sua vez, deverão ser legalmente obrigados a mudar a estrutura e a forma de reembolso dos serviços prestados pelos laboratórios de análises clínicas, pois o orçamento para gastos laboratoriais no setor primário deverá ser reduzi do em $20 \%$ em relação aos anos anteriores, como medida para evitar o déficit no orçamento dos Fundos (Müller, 1994). Tais medidas racionalizadoras, implementadas desde o início de 1994, vêm obrigando muitos laboratórios de pequenos hospitais a se associarem a laboratórios de grupo ou de institutos como estratégia de sobrevivência. Uma das conseqüências mais imediatas de tais medidas tem sido a relutância dos laboratórios 
em investir em novos equipamentos. No entanto, o resultado final de tais medidas só poderá ser avaliado posteriormente, principalmente após implementação das mudanças propostas para o setor hospitalar, a partir de 1996.

\section{O sistema de atenção hospitalar}

A maioria dos hospitais alemães é público ou privado de caráter não-lucrativo, de grande porte e abrangência regional, financiados sob regime orçamentário, cujo montante é negociado anualmente com os Fundos de Doença, sendo que os hospitais públicos contam também com contribuições orçamentárias federais, estaduais ou municipais, respectivamente, para hospitais mantidos pelo Governo Federal, Estadual ou Municipal (Kirkman, 1991). Em 1990, havia 1.816 hospitais gerais na Alemanha (antiga Ocidental), sendo que 727 eram públicos, 772 pertenciam à I greja ou a instituições de caráter público e apenas 317 eram privados, sendo que, destes, menos de $7 \%$ dos leitos eram lucrativos (Schulenburg, 1994). O orçamento hospitalar é o único caso de contrato não-coletivo no Sistema de Saúde alemão: é negociado caso a caso para cada um dos mais de 3.000 hospitais em funcionamento no país. Todos os funcionários de hospital recebem seus honorários através de salários, inclusive os médicos, sendo que os vários profissionais que ali atuam são classificados em categorias de acordo com a formação profissional e tipo de atuação como base para as negociações salariais. Assim, os médicos dos hospitais são remunerados através de salários, enquanto os que atuam em regime ambulatorial recebem por quantidade de serviços prestados, até que se institua o regime de captação, em 1996. O paciente tem a liberdade para escolher o médico de ambulatório e o hospital onde deseja ser atendido, mas não pode escolher o médico do hospital que irá atendê-lo.

Anualmente, os Fundos negociam com cada hospital os custos diários de cuidados de internação (custos dos cuidados de enfermagem mais custos dos exames laboratoriais) e os custos por paciente, estabelecendo o valor do orçamento para o ano seguinte, tendo por base o montante de recursos gastos nos anos anteriores (orçamento prospectivo calculado em bases retrospectivas). O cálculo do orçamento varia de acordo com o tamanho, a complexidade e os vários tipos de serviço prestados pelo hospital, sendo que a principal unidade de medida utilizada é a quantidade de “diárias hospitala- res" demandadas pelos filiados de um determinado Fundo junto àquele hospital, isto é, depende do número de pacientes que esteve ali internado e da média de permanência que apresentaram. Em 1992, o valor médio da diária hospitalar era de US\$223,00 por paciente, variando entre US\$120,00 e US\$600,00, dependendo do hospital (Müller, 1994). O valor das diárias a serem pagas pode variar bastante de um hospital a outro, e de um Fundo a outro, mas, em geral, todos el es estabelecem pagamento diferenciado, específico e pré-fixado para internações em Unidades de Terapia Intensiva e procedimentos de alta tecnologia, como cirurgias cardíacas e transplantes. Assim, os Fundos negociam o orçamento de cada hospital de acordo com o número e o tipo dos leitos ocupados por seus filiados, de modo que, quanto mais alta for a taxa de ocupação hospitalar, ou seja, quanto menor o número de leitos vazios durante o ano anterior, maior será o valor do orçamento de um dado hospital para o ano seguinte. Tal sistema tem sido um convite à manutenção de pacientes hospitalizados por longos períodos, tornando a média de permanência dos hospitais alemães exageradamente alta - em torno de 13,8 dias em 1990, variando de 5,7 dias para casos de otorrinolaringologia e chegando a 22,2 dias para doenças pulmonares (Schulenburg, 1994) - e deverá ser alterado no início de 1996, de acordo com as metas estabelecidas pelo GSG, implementado desde janeiro de 1993.

Por outro lado, além das altas médias de permanência, os hospitais alemães vêm apresentando uma demanda crescente de serviços que origina-se nas pressões exercidas, a partir da última reforma, sobre o setor ambulatorial de cuidados primários, o qual, muitas vezes, como estratégia para diminuir seus custos e riscos, aumenta o número de encaminhamentos para o setor hospitalar, assim como para os especialistas.

As medidas a serem implementadas em 1996 deixam de basear o cálculo do orçamento hospitalar em "diárias hospitalares" para fundamentarem-se na utilização de dois instrumentos associados: DRGs (Diagnosis Related Group) e PMCs (Patient Management Categories) (Schulenburg, 1994). A associação destes dois instrumentos, um relacionado ao custo médio de determinados grupos de patologias (DRG) e outro relacionado a categorias de conduta clínica (PMC), apesar da pouca disponibilidade de informações acerca de sua operacionalização, permite-nos supor que possam servir a propósitos semelhantes aos que se destinam dois instrumentos (Pink, 1994) em uso no 
Canadá, nas províncias de Ontário e Alberta : Case Mix Groups (CMG) e Resource Intensity Weights (RIW). Diferentemente da utilização isolada de DRGs, as quais permitem apenas que se estabeleçam mecanismos de pagamento para internações (baseados no cálculo do custo médio de internação para determinadas doenças ou grupos de doenças, de acordo com o diagnóstico de admissão, com a caracterização do procedimento como clínico ou cirúrgico, idade do paciente, complicações ou doenças correlatas e procedimentos demandados), a utilização associada de CMGs e RIWs viabiliza que se proceda a comparações acerca da performance de hospitais com características semelhantes ou com um mesmo porte de serviços, uma vez que permite a avaliação independente dos dois componentes: o clínico ou médico (CMG) e o financeiro (RIW), os quais, na DRG norte-americana, encontram-se agrupados (Pink, 1994).

O sistema CMG é, de certa forma, a versão canadense da DRG norte-americana, ou da AlH (Autorização para Internação Hospitalar) brasileira e fundamenta-se na 9a revisão do Código Internacional de Doenças (CID-9), condensando os mais de 10.000 códigos do CID-9 em 576 CM Gs, os quais se dividem em 25 grandes categorias clínicas (Pink, 1994). O sistema CMG fundamenta sua construção nos mesmos fatores da DRG, mas diferencia-se dela, na medida em que a caracterização de um paciente com um dado CM G serve-se de um algoritmo baseado no que os canadenses denominam "diagnóstico responsável", e que é determinado pela evolução clínica de um dado paciente, mais do que pelo diagnóstico de admissão, contemplando, portanto, uma diferenciação entre casos típicos e atípicos, ou entre evoluções favoráveis e desfavoráveis. A utilização de tais instrumentos viabiliza que a qualidade da atenção médica prestada possa atuar como importante fator interveniente na elaboração dos cálculos referentes à quantidade de recursos a ser recebida pelo hospital que prestou o atendimento. Sua associação com o sistema RIW viabiliza que os custos sejam estimados individualmente, de acordo com a evolução clínica de cada paciente, uma vez que o RIW constituise num instrumento que dimensiona a expectativa de utilização de recursos para cada CMG (Pink, 1994).

Estima-se que, com a implementação do sistema de DRG e PMC, ocorra uma diminuição no crescimento dos gastos hospitalares, os quais, em 1960, respondiam por $17,5 \%$ dos gastos do sistema e, em 1990, já demandavam $33,2 \%$ do orçamento dos Fundos, enquanto os gastos referentes a atenção ambulatorial passaram de 20,9\%, em 1960, para 18,2\%, em 1990 (Schulenburg, 1994).

A desvinculação entre o sistema de atenção hospitalar e a atenção ambulatorial tem gerado vários problemas, um dos quais tem sido a tendência em repetir, sob regime de internação, todos os exames feitos em ambulatório, assim como postergar a data da alta do paciente como medida de precaução, uma vez que o médico do hospital não pode garantir a qualidade do acompanhamento ambulatorial após a alta. A implementação de cirurgias ambulatoriais de menor custo e rápida recuperação pós-operatória tem sido dificultada, pois demandam ser realizadas fora do contexto hospitalar, implicando riscos que muitos médicos não querem assumir.

A qualidade dos serviços prestados pelos hospitais é sistematicamente monitorizada pelos Fundos de Doença, os quais podem romper contratos em vigor com hospitais que venham apresentando desempenho aquém do esperado, de acordo com os "padrões de boa conduta" ou "normas" ("guidelines") aceitos como adequados a determinados hospitais, dependendo do tipo e complexidade dos serviços hospitalares realizados. O estabelecimento de "padrões de boa conduta" ("guidelines") tem sido freqüente no Sistema de Saúde Alemão: a maioria dos contratos estabelece metas com relação à qualidade dos serviços prestados e negocia um consenso acerca dos parâmetros a serem utilizados para avaliar o desempenho dos distintos serviços ou ações sanitárias. Quando instituições ou serviços não obedecem ou não seguem os critérios de qualidade estabelecidos em seus contratos com os Fundos, estes podem ser rompidos com base em avaliações de desempenho, as quais são sistematicamente realizadas pelos Fundos, com relação aos hospitais, e pelas Associações Regionais de Médicos, com relação ao desempenho dos médicos que atuam na atenção ambulatorial.

Os gastos com hospitalização variam significativamente entre os Fundos, sendo que, entre 1965 e 1985, as taxas de admissão hospitalar dos Seguros-Saúde privados tiveram um crescimento de $30 \%$, a dos Fundos Substitutivos tiveram um aumento de $48 \%$ e a dos outros Fundos de Doença cresceram 73\%, denotando profundas diferenças, não apenas nos gastos e nos serviços demandados pelos vários Fundos, como apontando prováveis e significativas diferenças no perfil de morbidade da população segurada (Wysong, 1990). 
Algumas considerações sobre a o peracionalização do sistema

A Alemanha tem o maior contingente de médicos per capita entre os países do primeiro mundo, sendo que, em 1970, tinha 1,69 médicos/ 1.000 habitantes, passando a 2,21/ $1000 \mathrm{em}$ 1980, e chegando a 3,11 médicos/ 1.000 habitantes em 1990 (Henke, 1994). A constituição alemã não permite que se restrinja o acesso à escola médica (limitado apenas pela performance do aluno durante o curso do segundo grau), a qual apresenta-se popular por ser subsidiada pelo governo e por serem os médicos muito respeitados e terem grande status nas comunidades. Entre 1977 e 1989, o número de médicos atuantes no sistema ambulatorial cresceu $31 \%$, sem um correspondente aumento no número de pessoas seguradas (Roy, 1993). Deste modo, algumas estratégias têm sido utilizadas para limitar o número de médicos contratados por Fundo de Doença, como o estabelecimento de limites em relação à especialidade médica, a imposição de restrições ou incentivos acerca da região em que o médico irá atuar, de acordo com as necessidades do sistema. Uma das medidas explícitas para diminuir o contingente de médicos em atuação na Alemanha, e que deverá ser implementada em 1999, refere-se à revogação das licenças dos médicos com mais de 68 anos de idade, e que venham atuando no sistema há mais de 20 anos.

Os fundos estabel ecem limites orçamentarios diferentes para as distintas atividades executadas, as quais, de uma maneira geral, apresentam a seguinte proporcionalidade, segundo Shulenburg (1994):

\begin{tabular}{lr}
\hline Atenção primária & $18,2 \%$ \\
Atenção odontológica/próteses & $9,7 \%$ \\
Medicamentos & $16,3 \%$ \\
Hospitalização & $33,2 \%$ \\
Outros gastos & $17,5 \%$ \\
Custos administrativos & $5,1 \%$ \\
\hline
\end{tabular}

Podemos observar que o Sistema de Saúde Alemão apresenta um custo administrativo bastante baixo em relação à maioria dos outros Sistemas do mundo, sendo, no entanto, aventado que poderiam aumentar caso a competição entre os Fundos se acentuasse, fato este bastante improvável com a implementação da ECR. O sistema estabelece limites explícitos para determinados gastos, como, por exemplo, determinando que apenas $10,5 \%$ do montante de recursos referente a honorários médicos da atenção primária poderiam ser destinados ao pagamento de atividades caracterizadas como procedimentos técnicos (exames laboratoriais ou radiológicos), enquanto $47,5 \%$ deveriam destinar-se a honorários referentes a contatos pessoais, como consultas e exames clínicos, sendo o restante destinado a cuidados preventivos (Powell, 1994). Tais medidas, segundo Müller (1994), têm sido relacionadas à diminuição do número de médicos em atuação nos laboratórios de MG.

Como medida de incentivo à realização de determinados procedimentos ambulatoriais, o Sistema estabelece uma pontuação graduada específica para determinados conjuntos de procedimentos técnicos realizados, a qual tem relação direta com o montante de recursos destinado a financiar tais procedimentos, permitindo, por exemplo, que se privilegiem serviços básicos em relação aos especializados. Assim, viabiliza que o montante a ser pago dependa não apenas do volume de procedimentos realizados, mas também da relevância ou importância de um dado procedimento junto ao sistema como um todo. No entanto, longe de ser arbitrária, tal pontuação é estabelecida mediante prévia e ampla discussão entre os Fundos de Doença e as Associações de Médicos, no sentido de privilegiar com uma maior pontuação as atividades consideradas mais relevantes.

Em 1993, tal sistema de pontuação foi modificado para aumentar a remuneração referente a consultas, exame físico e cuidados preventivos nos serviços de atenção primária, mas suas conseqüências têm se mostrado pouco significativas, incorrendo num maior número de encaminhamentos para especialistas e internações hospitalares por parte dos generalistas, que se vêem ameaçados pela possibilidade de queixas de pacientes, os quais podem acusá-los de negligência por não terem indicado a realização de determinados exames diagnósticos (Henke, 1994; Bousigen, 1995).

Henke (1994) relata que a mudança na pontuação dos procedimentos não tem apresentado impacto significativo na renda dos médicos alemães, inclusive não havendo indícios de uma mudança no padrão de atuação destes, no sentido de um maior empenho na execução de atividades preventivas. No entanto, pesquisa (Hoopmann, 1995) realizada na região da Saxônia, durante o ano de 1993, aponta a ocorrência de mudanças no padrão de atuação médica, principalmente no que se refere ao atendimento de pacientes com queixas e/ ou sintomas sugestivos de doenças que contemplam forte componente emocional, sendo que os médicos participantes da pesquisa responsabi- 
lizaram, em grande parte, as medidas de contenção de custos como as principais responsáveis pelas mudanças ocorridas em seu padrão de conduta. Tais medidas de contenção de custos, segundo os médicos entrevistados, não causaram diminuição na qualidade dos serviços prestados, mas têm sido por eles percebidas como geradoras de uma maior insatisfação dos pacientes para com os cuidados recebidos, uma vez que os pacientes desejam, muitas vezes, submeter-se a procedimentos que são avaliados pelo sistema, assim como pelos médicos, como não-fundamentais, ou com uma baixa relação custo-benefício em determinados contextos clínicos específicos (Hoopmann, 1995).

Esta tendência de comportamento também foi detectada em nosso meio, onde estudo (Guia, 1995) realizado na cidade de São Paulo aponta que os pacientes tendem a avaliar a competência médica através da prescrição, ou não, de determinados procedimentos diagnósticos ou terapêuticos que se servem de "tecnologia de ponta”, a qual é por eles percebida como associada à obtenção de melhores resultados clínicos.

Medidas para o incentivo de cirurgias ambulatoriais estabeleciam que os gastos com tais procedimentos poderiam crescer $10 \%$ a mais do que o permitido pela arrecadação dos Fundos, assim como os cuidados preventivos poderiam demandar $6 \%$ mais recursos que os curativos, sendo que, tais medidas, implementadas previamente às mudanças no sistema hospitalar, vêm apresentando pouco impacto (Henke, 1994).

O Sistema de Saúde Alemão exerce rígida e sistemática fiscalização sobre os serviços de saúde, particularmente os executados pelos médicos. As prescrições de medicamentos, indicações de exames e mesmo internações são controladas através do estabelecimento de parâmetros para avaliação dos vários padrões de atuação esperados em cada nível de atenção e especialidade. Tais parâmetros são estabelecidos mediante ampla negociação entre as Associações de Médicos e os Fundos de Doença, sendo definidos conjuntamente vários graus de penalidades para cada "desvio" detectado em relação aos padrões estabelecidos, os quais variam de uma monitorização mais detalhada da performance de um dado profissional até a devolução de pagamentos recebidos e avaliados como decorrentes de desempenho tecnicamente inadequado. Henke relata que, anualmente, cerca de 7\% dos médicos alemães são chamados a dar explicações sobre suas condutas clínicas, mas que apenas $2 \%$ deles necessi- tam proceder a devoluções dos recursos recebidos (Henke, 1994).

\section{Comentários finais}

Há consenso de que o propósito da maioria das mudanças implementadas na Alemanha, desde o final dos anos 70, não tenha sido o estabelecimento de prioridades sanitárias (Von Stillfried, 1993), mas sim o de tornar os cuidados a saúde o mais barato possível, pois os médicos e os Fundos, temerosos de ações legais, não têm estabelecido limites explícitos no uso de tecnologias, sendo que a solução que se lhes apresenta tem sido barateá-las, e não restringir explicitamente sua utilização. No entanto, apesar de os gastos do Sistema estarem se mantendo estáveis em relação ao PIB, o total de gastos demandado por pessoa segurada vem aumentando (Von Stillfried, 1993), denotando as várias limitações das medidas até então implementadas. No entanto, al gumas lições podem ser apreendidas a partir da experiência alemã, primeira e principalmente com vistas à efetiva implementação do nosso Sistema único de Saúde.

A primeira delas é que o setor privado pode ser um parceiro eficiente quando operacionalizado sob diretrizes políticas sérias e claras, onde as competências, direitos e obrigações, quando não cumpridas sob a forma da lei, são passíveis de punições exemplares em todo e qualquer nível do sistema sanitário.

A segunda é que medidas controladoras e sanções explícitas, mesmo quando altamente impopulares entre os médicos, são passíveis de serem aplicadas e viabilizadas, quando fundamentadas em dados contundentes e objetivos coletados através de um sistema de informações confiável e implementadas mediante amplo processo de negociação entre os envolvidos. Assim, o estabelecimento de parâmetros avaliatórios pertinentes à realidade local faz-se fundamental e imprescindível a um desempenho minimamente satisfatório, seja do ponto de vista econômico ou sanitário, em qualquer Sistema de Saúde, demandando a disponibilidade de critérios explícitos para a utilização e avaliação do desempenho das inúmeras novas ou inovadas tecnologias médicas que incorporamos sistematicamente aos nossos serviços de saúde. Um exemplo pode ser apreendido através do processo de reforma implementado, em 1990, no National Health Service (NHS), na Inglaterra. A escassa disponibilidade de parâmetros e informações (Maynard, 1994) sobre o desempenho das várias instituições de saúde 
que compõem o NHS tem dificultado enormemente o estabelecimento de uma competição entre os vários provedores de serviços de saúde (particularmente os Trusts - hospitais públicos com autonomia de gestão) (Ovretveit, 1993), uma vez que não tem sido possível avaliar, com segurança, quais são as instituições que produzem serviços de melhor qualidade e com uma relação custo-benefício mais vantajosa. O conceito de competição de mercado de Alain Enthoven (Enthoven, 1994), que forneceu as premissas para a reforma do NHS, somente pode ser viabilizado se houver disponibilidade de um sistema de informações seguro e fidedigno que possibilite o desenvolvimento de parâmetros de avaliação, no sentido de viabilizar o estabelecimento de uma competitividade baseada não apenas nos preços, como também na qualidade dos serviços prestados e nos resultados obtidos pelos pacientes no tocante a sua situação de saúde. Assim, a implementação do processo de reforma antes do desenvolvimento de um sistema de informações que pudesse fornecer bases sólidas para o estabelecimento da competição de mercado tem sido apontada como uma das principais causas da lentidão com que vem se processando a reforma do Sistema de Saúde inglês.

A terceira, e mais importante delas, referese a uma ocorrência de natureza macrossocial que viabiliza uma associação entre "alta resolubilidade" ou "obtenção resultados altamente satisfatórios" com a utilização de vasto e sofisticado arsenal científico-tecnológico. Tal tendência, identificada por Tornatzky (Tornatzky, 1990) como um "viés pró-inovação", onde as novidades científico-tecnológicas são consumidas como significantes lineares de um meIhor desempenho institucional ou profissional, reflete-se nos Sistemas de Saúde da sociedade ocidental contemporânea, permitindo que pacientes se sintam insatisfeitos com os cuidados recebidos e médicos se apresentem inseguros de sua prática quando esta não se apóia no vasto arsenal da propedêutica armada ou em sofisticadas tecnologias terapêuticas. Podemos perceber que as medidas implementadas na Alemanha destinam-se a diminuir o impacto do que podemos denominar de "imperativo para consumo de tecnologias médicas" (Guia, 1995). No entanto, sabemos que o potencial de inúmeras, sofisticadas e caras tecnologias médicas "de ponta" em proporcionar uma maior expectativa de vida ou uma melhor qualidade de vida, no que se refere à saúde das comunidades humanas, permanece por ser demonstrado, apesar de se nos apresentar como potencialmente benéfico, a nível individual. A estra- tégia alemã tenta enfrentar uma contradição que vem acompanhando a prática médica ocidental contemporânea, e que emerge como um problema a ser resolvido na maioria dos sistemas de saúde ocidentais: criamos, valorizamos e incentivamos a utilização de vasto e sofisticado arsenal médico-tecnológico e, quando percebemos que não conseguimos "pagar a conta" demandada por esta medicina especializada e altamente tecnificada, criamos mecanismos paralelos, no sentido de incentivar a utilização de outras práticas, como as caracterizadas por um "maior conteúdo relacional" ou "mais humanísticas e menos tecnicistas", as quais constituem-se não apenas em estratégias menos onerosas, como, em não raras vezes, apresentam resultados mais satisfatórios.

A solução de problemas desta natureza, no entanto, demanda mudanças muito mais amplas e profundas, em inúmeros outros setores da sociedade, e não apenas nos Sistemas de Saúde. Remete aos valores que construíram e sustentam a sociedade capitalista e industrial do Ocidente, os quais incentivam altos investimentos financeiros no desenvolvimento das mais variadas, caras e sofisticadas tecnologias médicas, as quais alimentam lucros fabulosos com a esperança humana de uma vida melhor e mais saudável. 


\section{Referências}

BRENNER, G. \& RUBLEE, D. A., 1991. The 1987 revision of physician fees in Germany. Health Affairs, 10:147-156.

BOUSIGEN, D. D., 1995. Maîtrise comptable: les limites de l'exemple allemand. Quotidien du Médicin, 5721:5725.

ENTHOVEN, A. C., 1994. On the ideal market structure for thirdy-party purchasing of health care. Social Science and Medicine, 39:1413-1424.

GUIA, R. G. P., 1995. O Elo Partido: O Relacionamento Médico-Pacientena Era Tecnológica. Dissertação de Mestrado, São Paulo: Faculdade de Saúde Pública da Universidade de São Paulo.

HENKE, K. D.; MURRAY, M. A. \& ADE, C., 1994. Global budgeting in Germany: the lessons for the United States. Health Affairs, 13:7-21.

HOOPMANN, M.; SCHWARTZ, F. W. \& WEBER, J., 1995. Effects of the German 1993 health reform law upon primary care practitioners' individual performance: results from an empirical study sentinel practices. Journal of Epidemiology and Community Health, 49 (suppl):33-36.

KIRKM AN, B. L., 1991. Health insurance values and implementation in the Netherlands and the Republic of Germany. Journal of American Medical Association, 265:2496-2502.

MAYNARD, A., 1994. Can competition enhance efficiency in health care? Lessons from the reform of the U.K. National Health Service. Social Science and Medicine, 39:1433-1445.

MÜLLER, B., 1994. Laboratory diagnostics in light of massive changes in official health policies. Clinical Chemistry, 40:1658-1662.

OMS (Organización Mundial de la Salud), 1992. Reformas de los sistemas sanitarios en Europa. Primera Reunión del Grupo de Trabajo sobre la Reforma de los Sistemas Sanitarios en Europa. Madrid: Oficina Regional para Europa.

OVRETVEIT, J., 1993. Purchasing for health gain: the problems and prospects for purchasing for health gain in the 'managed markets' of the NHS and other European health systems. European Journal of Public Health, 3:77-84.
PINK, G. H. \& BOLLEY, H. B., 1994. Physicians in health care management: 3. Case Mix Groups and Resource Intensity Weights: an overview for physicians.Canadian Medical Association Journal, 150:889-894.

PINK,G. H. \& BOLLEY, H. B., 1994. Physicians in health care management: 4. Case Mix Groups and Resource Intensity Weights: physicians and hospital funding. Canadian Medical Association Journal, 150:1255-1261.

POWELL, F. D., 1994. Government participation in physician negotiations in German economic policy as applied to universal health care coverage in The United States. Social Science and Medicine, 38:35-43.

REINHARDT, U. E., 1994. Germany's Health Care System: it's not the american way. Health Affairs, 13:22-24.

ROGAL, D. L.; GAUTHIER, A. K. \& BARRAND, N. L., 1993. Managing the Health Care System under a global expenditure limit: a workshop summary. Inquiry, 30:318-322.

ROY, M., 1993. The German Health Care System: model or mirage? Southern Medical Journal, 86:13891394.

SCHULENBURG, J. M. G., 1994. Forming and reforming the market for thirdy-party purchasing of health care: a german perspective. Social Science and Medicine, 39:1473-1481.

TORNATZKY, L. G., 1990. The Processes of Technological Innovation. Massachusetts and Toronto: Lexington Books.

VON STILLFRIED, D. \& ARNOLD, M., 1993. What's happening to health care in Germany? British Me dical Journal, 306:1017-1018.

WYSONG, J. A. \& ABEL, T., 1990. Universal Health Insurance and high-risk groups in West Germany: implications for U.S. health policy. Milbank Quarterly, 68:527-561. 